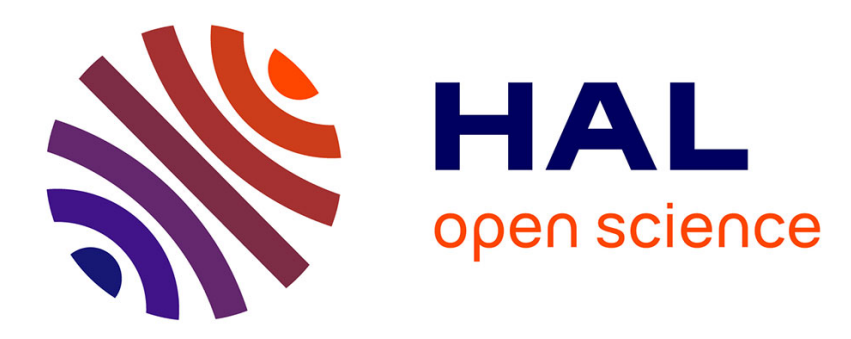

\title{
Regularizing Common Spatial Patterns to Improve BCI Designs: Unified Theory and New Algorithms \\ Fabien Lotte, Cuntai Guan
}

\section{To cite this version:}

Fabien Lotte, Cuntai Guan. Regularizing Common Spatial Patterns to Improve BCI Designs: Unified Theory and New Algorithms. IEEE Transactions on Biomedical Engineering, 2011, 58 (2), pp.355-362. inria-00476820v4

\section{HAL Id: inria-00476820 https://hal.inria.fr/inria-00476820v4}

Submitted on 24 Sep 2010

HAL is a multi-disciplinary open access archive for the deposit and dissemination of scientific research documents, whether they are published or not. The documents may come from teaching and research institutions in France or abroad, or from public or private research centers.
L'archive ouverte pluridisciplinaire HAL, est destinée au dépôt et à la diffusion de documents scientifiques de niveau recherche, publiés ou non, émanant des établissements d'enseignement et de recherche français ou étrangers, des laboratoires publics ou privés. 


\title{
Regularizing Common Spatial Patterns to Improve BCI Designs: Unified Theory and New Algorithms
}

\author{
Fabien LOTTE*, Member, IEEE, Cuntai GUAN, Senior Member, IEEE
}

\begin{abstract}
One of the most popular feature extraction algorithms for Brain-Computer Interfaces (BCI) is Common Spatial Patterns (CSP). Despite its known efficiency and widespread use, CSP is also known to be very sensitive to noise and prone to overfitting. To address this issue, it has been recently proposed to regularize CSP. In this paper, we present a simple and unifying theoretical framework to design such a Regularized CSP (RCSP). We then present a review of existing RCSP algorithms, and describe how to cast them in this framework. We also propose 4 new RCSP algorithms. Finally, we compare the performances of 11 different RCSP (including the 4 new ones and the original CSP), on EEG data from 17 subjects, from BCI competition data sets. Results showed that the best RCSP methods can outperform CSP by nearly $10 \%$ in median classification accuracy and lead to more neurophysiologically relevant spatial filters. They also enable us to perform efficient subject-to-subject transfer. Overall, the best RCSP algorithms were CSP with Tikhonov Regularization and Weighted Tikhonov Regularization, both proposed in this paper.
\end{abstract}

Index Terms-brain-computer interfaces (BCI), EEG, common spatial patterns (CSP), regularization, subject-to-subject transfer

\section{INTRODUCTION}

Brain-Computer Interfaces (BCI) are communication systems which enable users to send commands to computers by using brain activity only, this activity being generally measured by ElectroEncephaloGraphy (EEG) [1]. BCI are generally designed according to a pattern recognition approach, i.e., by extracting features from EEG signals, and by using a classifier to identify the user's mental state from such features [1][2]. The Common Spatial Patterns (CSP) algorithm is a feature extraction method which can learn spatial filters maximizing the discriminability of two classes [3][4]. CSP has been proven to be one of the most popular and efficient algorithms for BCI design, notably during BCI competitions [5][6].

Despite its popularity and efficiency, CSP is also known to be highly sensitive to noise and to severely overfit with small training sets [7][8]. To address these drawbacks, a recent idea has been to add prior information into the CSP learning process, under the form of regularization terms [9][10][11][12] (see Section IV-A for a review). These Regularized CSP (RCSP) have all been shown to outperform classical CSP. However, they are all expressed with different formulations and therefore lack a unifying regularization framework. Moreover, they were only compared to standard CSP, and typically with 4 or 5 subjects only [9][10][11], which makes it difficult to assess their relative performances. Finally, we believe that a variety of other priors could be incorporated into CSP.

F. Lotte and C. Guan are with the Institute for Infocomm Research, $1 \mathrm{Fu}-$ sionopolis Way, 138632, Singapore. e-mail: fprlotte,ctguan@i2r.a-star.edu.sg
Therefore, in this paper we present a simple theoretical framework that could unify RCSP algorithms. We present existing RCSP within this unified framework as well as 4 new RCSP algorithms, based on new priors. It should be mentioned that preliminary studies of 2 of these new algorithms have been presented in conference papers [12][13]. Finally, we compare these various algorithms on EEG data from 17 subjects, from publicly available BCI competition data sets.

This paper is organized as follows: Section II describes the CSP algorithm while Section III presents the theoretical framework to regularize it. Section IV expresses existing RCSP within this framework and presents 4 new RCSP. Finally, Sections V and VI describe the evaluations performed and their results, and conclude the paper, respectively.

\section{THE CSP ALGORITHM}

CSP aims at learning spatial filters which maximize the variance of band-pass filtered EEG signals from one class while minimizing their variance from the other class [4][3]. As the variance of EEG signals filtered in a given frequency band corresponds to the signal power in this band, CSP aims at achieving optimal discrimination for BCI based on band power features [3]. Formally, CSP uses the spatial filters $w$ which extremize the following function:

$$
J(w)=\frac{w^{T} X_{1}^{T} X_{1} w}{w^{T} X_{2}^{T} X_{2} w}=\frac{w^{T} C_{1} w}{w^{T} C_{2} w}
$$

where $T$ denotes transpose, $X_{i}$ is the data matrix for class $i$ (with the training samples as rows and the channels as columns) and $C_{i}$ is the spatial covariance matrix from class $i$, assuming a zero mean for EEG signals. This last assumption is generally met when EEG signals are band-pass filtered. This optimization problem can be solved (though this is not the only way) by first observing that the function $J(w)$ remains unchanged if the filter $w$ is rescaled. Indeed $J(k w)=J(w)$, with $k$ a real constant, which means the rescaling of $w$ is arbitrary. As such, extremizing $J(w)$ is equivalent to extremizing $w^{T} C_{1} w$ subject to the constraint $w^{T} C_{2} w=1$ as it is always possible to find a rescaling of $w$ such that $w^{T} C_{2} w=1$. Using the Lagrange multiplier method, this constrained optimization problem amounts to extremizing the following function:

$$
L(\lambda, w)=w^{T} C_{1} w-\lambda\left(w^{T} C_{2} w-1\right)
$$

The filters $w$ extremizing $L$ are such that the derivative of $L$ with respect to $w$ equals 0 :

$$
\begin{aligned}
\frac{\partial L}{\partial w} & =2 w^{T} C_{1}-2 \lambda w^{T} C_{2}=0 \\
& \Leftrightarrow C_{1} w=\lambda C_{2} w \\
& \Leftrightarrow \quad C_{2}^{-1} C_{1} w=\lambda w
\end{aligned}
$$


We obtain a standard eigenvalue problem. The spatial filters extremizing Eq. 1 are then the eigenvectors of $M=C_{2}^{-1} C_{1}$ which correspond to its largest and lowest eigenvalues. When using CSP, the extracted features are the logarithm of the EEG signal variance after projection onto the filters $w$.

\section{REgulARIZED CSP: THEORY}

As mentioned above, to overcome the sensitivity of CSP to noise and overfitting, one should regularize it. Adding prior information to CSP, and thus regularizing it, can be done at two levels. First, it can be done at the covariance matrix estimation level. Indeed, CSP relying on covariance matrix estimates, such estimates can suffer from noise or small training sets, and thus benefit from regularization. Another approach consists in regularizing CSP at the level of the objective function (Eq. 1), by imposing priors on the spatial filters to obtain. The remaining of this section presents these two approaches.

\section{A. Regularizing the covariance matrix estimates}

CSP requires to estimate the spatial covariance matrix for each class. However, if the EEG training set is noisy and/or small, these covariance matrices may be poor or nonrepresentative estimates of the mental states involved, and thus lead to poor spatial filters. Therefore, it is appropriate to add prior information to these estimates by using regularization terms. Based on [10], it can be performed as follows:

$$
\begin{aligned}
\tilde{C}_{c} & =(1-\gamma) \hat{C}_{c}+\gamma I \\
\text { with } \hat{C}_{c} & =(1-\beta) s_{c} C_{c}+\beta G_{c}
\end{aligned}
$$

where $C_{c}$ is the initial spatial covariance matrix for class $c, \tilde{C}_{c}$ is the regularized estimate, $I$ is the identity matrix, $s_{c}$ is a constant scaling parameter (a scalar), $\gamma$ and $\beta$ are two user-defined regularization parameters $(\gamma, \beta \in[0,1])$ and $G_{c}$ is a "generic" covariance matrix (see below). Two regularization terms are involved here. The first one, associated to $\gamma$, shrinks the initial covariance matrix estimate towards the identity matrix, to counteract a possible estimation bias due to a small training set. The second term, associated to $\beta$, shrinks the initial covariance matrix estimate towards a generic covariance matrix, to obtain a more stable estimate. This generic matrix represents a given prior on how the covariance matrix for the mental state considered should be. This matrix is typically built by using signals from several subjects whose EEG data has been recorded previously. This has been shown to be an effective way to perform subject-to-subject transfer [11][10][13]. However, it should be mentioned that $G_{c}$ could also be defined based on neurophysiological priors only.

Learning spatial filters with this method simply consists in replacing the covariance matrices $C_{1}$ and $C_{2}$ used in CSP by their regularized estimates $\tilde{C}_{1}$ and $\tilde{C}_{2}$. Many different RCSP algorithms can be thus designed, depending on whether one or both regularization terms are used, and more importantly, on how the generic covariance matrix $G_{c}$ is built.

\section{B. Regularizing the CSP objective function}

Another approach to obtain regularized CSP algorithms consists in regularizing the CSP objective function itself (Eq. 1). More precisely, such a method consists in adding a regularization term to the CSP objective function in order to penalize solutions (i.e., resulting spatial filters) that do not satisfy a given prior. Formally, the objective function becomes:

$$
J_{P_{1}}(w)=\frac{w^{T} C_{1} w}{w^{T} C_{2} w+\alpha P(w)}
$$

where $P(w)$ is a penalty function measuring how much the spatial filter $w$ satisfies a given prior. The more $w$ satisfies it, the lower $P(w)$. Hence, to maximize $J_{P_{1}}(w)$, we must minimize $P(w)$, thus ensuring spatial filters satisfying the prior. $\alpha$ is a user-defined regularization parameter $(\alpha \geq 0$, the higher $\alpha$, the more satisfied the prior). With this regularization, we expect that enforcing specific solutions, thanks to priors, will guide the optimization process towards good spatial filters, especially with limited or noisy training data.

In this paper, we focus on quadratic penalties: $P(w)=$ $\|w\|_{K}^{2}=w^{T} K w$, where matrix $K$ encodes the prior. Interestingly enough, RCSP with non-quadratic penalties have been proposed [14][15]. They used an $l_{1}$ norm penalty to select a sparse set of channels. However, these studies showed that sparse CSP generally gave lower performances than CSP (with all channels), although they require much less channels, hence performing efficient channel reduction. As the focus of this paper is not channel reduction but performance enhancement, we only consider quadratic penalties here. Moreover, quadratic penalties lead to a close form solution for optimization (see below), which is more convenient and computationally efficient. With a quadratic penalty term, Eq. 5 becomes:

$$
\begin{aligned}
J_{P_{1}}(w) & =\frac{w^{T} C_{1} w}{w^{T} C_{2} w+\alpha w^{T} K w} \\
& =\frac{w^{T} C_{1} w}{w^{T}\left(C_{2}+\alpha K\right) w}
\end{aligned}
$$

The corresponding Lagrangian is:

$$
L_{P_{1}}(\lambda, w)=w^{T} C_{1} w-\lambda\left(w^{T}\left(C_{2}+\alpha K\right) w-1\right)
$$

By following the same approach as previously (see Section II), we obtain the following eigenvalue problem:

$$
\left(C_{2}+\alpha K\right)^{-1} C_{1} w=\lambda w
$$

Thus, the filters $w$ maximizing $J_{P_{1}}(w)$ are the eigenvectors corresponding to the largest eigenvalues of $M_{1}=\left(C_{2}+\right.$ $\alpha K)^{-1} C_{1}$. With CSP, the eigenvectors corresponding to both the largest and smallest eigenvalues of $M$ (see Section II) are used as the spatial filters, as they respectively maximize and minimize Eq. 1 [4]. However, for RCSP, the eigenvectors corresponding to the lowest eigenvalues of $M_{1}$ minimize Eq. 5 , and as such maximize the penalty term (which should be minimized). Therefore, in order to obtain the filters which maximize $C_{2}$ while minimizing $C_{1}$, we also need to maximize the following objective function:

$$
J_{P_{2}}(w)=\frac{w^{T} C_{2} w}{w^{T} C_{1} w+\alpha P(w)}
$$


which is achieved by using the eigenvectors corresponding to the largest eigenvalues of $M_{2}=\left(C_{1}+\alpha K\right)^{-1} C_{2}$ as the filters $w$. In other words, with RCSP, the spatial filters used are the eigenvectors corresponding to the largest eigenvalues of $M_{1}$ and to the largest eigenvalues of $M_{2}$. With this approach, various regularized CSP algorithms can be designed depending on the knowledge encoded into matrix $K$.

\section{Summary}

We have presented two theoretical approaches to design RCSP algorithms: one at the covariance matrix estimation level and one at the objective function level. Naturally, these two approaches are not exclusive and can be combined within the same framework. Table I summarizes this framework and highlights the differences between CSP and RCSP. With this framework, many different RCSP can be designed depending on 1) which of the 3 regularization terms (associated to $\alpha, \beta$ and $\gamma$ ) is (are) used and on 2) how the matrices $G_{c}$ and $K$ are built. The following section presents several such variants, including existing algorithms as well as 4 new ones.

TABLE I

DIFFERENCES IN OBJECTIVE FUNCTION AND ALGORITHM OPTIMIZATION BETWEEN A STANDARD CSP AND A REGULARIZED CSP (RCSP).

\begin{tabular}{|c|c|c|}
\hline & CSP & RCSP \\
\hline \multirow{6}{*}{$\begin{array}{l}\text { Objective } \\
\text { function }\end{array}$} & $w^{T} C_{1} w$ & $w^{T} \tilde{C}_{\{1,2\}} w$ \\
\hline & $J(w)=\overline{w^{T} C_{2} w}$ & $J_{\{1,2\}}(w)=\overline{w^{T} \tilde{C}_{\{2,1\}} w+\alpha P(w)}$ \\
\hline & & with \\
\hline & & $P(w)=w^{T} K w$ \\
\hline & & $\tilde{C}_{c}=(1-\gamma) \hat{C}_{c}+\gamma I$ \\
\hline & & $\hat{C}_{c}=(1-\beta) s_{c} C_{c}+\beta G_{c}$ \\
\hline \multirow{5}{*}{$\begin{array}{c}\text { Solutions } \\
\text { of the } \\
\text { optimization } \\
\text { problem }\end{array}$} & eigenvectors & eigenvectors corresponding \\
\hline & corresponding & to the $N_{f}$ largest \\
\hline & to the $N_{f}$ largest & eigenvalues of \\
\hline & and $N_{f}$ lowest & $M_{1}=\left(\tilde{C}_{2}+\alpha K\right)^{-1} \tilde{C}_{1}$ \\
\hline & of $M=C_{2}^{-1} C_{1}$ & $M_{2}=\left(\tilde{C}_{1}+\alpha K\right)^{-1} \tilde{C}_{2}$ \\
\hline
\end{tabular}

\section{Regularized CSP: Algorithms}

\section{A. Existing RCSP algorithms}

Four RCSP algorithms have been proposed so far: Composite CSP, Regularized CSP with Generic Learning, Regularized CSP with Diagonal Loading and invariant CSP. They are described below within the presented framework.

\section{1) Composite CSP:}

The Composite CSP (CCSP) algorithm, proposed by Kang et al [11], aims at performing subject-to-subject transfer by regularizing the covariance matrices using other subjects' data. Expressed within the framework of this paper, CCSP uses only the $\beta$ hyperparameter $(\alpha=\gamma=0)$, and defines the generic covariance matrices $G_{c}$ according to covariance matrices of other subjects. Two methods were proposed to build $G_{c}$.

With the first method, denoted here as CCSP1, $G_{c}$ is built as a weighted sum of the covariance matrices (corresponding to the same mental state) of other subjects, by de-emphasizing covariance matrices estimated from fewer trials:

$$
G_{c}=\sum_{i \in \Omega} \frac{N_{c}^{i}}{N_{t, c}} C_{c}^{i} \quad \text { and } \quad s_{c}=\frac{N_{c}}{N_{t, c}}
$$

where $\Omega$ is a set of subjects whose data is available, $C_{c}^{i}$ is the spatial covariance matrix for class $c$ and subject $i, N_{c}^{i}$ is the number of EEG trials used to estimate $C_{c}^{i}, N_{c}$ is the number of EEG trials used to estimate $C_{c}$ (matrix for the target subject), and $N_{t, c}$ is the total number of EEG trials for class $c$ (from all subjects in $\Omega$ together with the target subject).

With the second method, denoted as CCSP2, $G_{c}$ is still a weighted sum of covariance matrices from other subjects, but the weights are defined according to the Kullback-Leibler (KL) divergence between subjects' data:

$$
G_{c}=\sum_{i \in \Omega} \frac{1}{Z} \frac{1}{K L(i, t)} C_{c}^{i} \quad \text { with } \quad Z=\sum_{j \in \Omega} \frac{1}{K L(j, t)}
$$

where $K L(i, t)$ is the KL-divergence between the target subject $t$ and subject $i$, and is defined as follows:

$$
K L(i, t)=\frac{1}{2}\left(\log \left(\frac{\operatorname{det}\left(C_{c}\right)}{\operatorname{det}\left(C_{c}^{i}\right)}\right)+\operatorname{tr}\left(C_{c}^{-1} C_{c}^{i}\right)-N_{e}\right)
$$

where det and $\operatorname{tr}$ are respectively the determinant and the trace of a matrix, and $N_{e}$ is the number of electrodes used. With CCSP2, the scaling constant $s_{c}$ is equal to 1 .

2) Regularized CSP with Generic Learning:

The RCSP approach with Generic Learning, proposed by $\mathrm{Lu}$ et al [10] and denoted here as GLRCSP, is another approach which aims at regularizing the covariance matrix estimation using data from other subjects. GLRCSP uses both the $\beta$ and $\gamma$ regularization terms, i.e., it aims at shrinking the covariance matrix towards both the identity matrix and a generic covariance matrix $G_{c}$. Similarly to CCSP, $G_{c}$ is here computed from the covariance matrices of other subjects such that $G_{c}=s_{G} \sum_{i \in \Omega} C_{c}^{i}$, where $s_{c}=s_{G}=\frac{1}{(1-\beta) M_{C_{c}}+\beta \sum_{i \in \Omega} M_{C_{c}^{i}}}$, and $M_{C}$ is the number of trials used to compute the covariance matrix $C$.

\section{3) Regularized CSP with Diagonal Loading:}

Another form of covariance matrix regularization used in the BCI literature is Diagonal Loading (DL), which consists in shrinking the covariance matrix towards the identity matrix. Thus, this approach only uses the $\gamma$ regularization parameter ( $\beta=\alpha=0$ ). Interestingly enough, in this case the value of $\gamma$ can be automatically identified using Ledoit and Wolf's method [16]. We denote this RCSP based on automatic DL as DLCSPauto. In order to check the efficiency of this automatic regularization for discrimination purposes, we will also investigate a classical selection of $\gamma$ using crossvalidation. We denote the resulting algorithm as DLCSPcv. When using Ledoit and Wolf's method for automatic regularization, the value of $\gamma$ selected to regularize $C_{1}$ can be different than that selected to regularize $C_{2}$. Therefore, we also investigated cross-validation to select a potentially different regularization parameter for $C_{1}$ and $C_{2}$. We denote this method as DLCSPcvdiff. To summarize, DLCSPauto automatically selects two $\gamma$ regularization parameters (one for $C_{1}$ and one for $C_{2}$ ) ; DLCSPcv selects a single $\gamma$ regularization parameter for both $C_{1}$ and $C_{2}$ using cross validation ; finally, DLCSPcvdiff selects two $\gamma$ regularization parameters (one for $C_{1}$ and one for $C_{2}$ ) using cross 
validation. It should be mentioned that, although covariance matrix regularization based on DL has been used in the BCI literature (see, e.g., [17]), to our best knowledge, it has not been used for CSP regularization, but for regularization of other algorithms such as Linear Discriminant Analysis (LDA).

\section{4) Invariant CSP:}

Invariant CSP (iCSP), proposed by Blankertz et al [9], aims at regularizing the CSP objective function in order to make filters invariant to a given noise source (it uses $\beta=\gamma=0$ ). To do so, the regularization matrix $K$ is defined as the covariance matrix of this noise source, e.g., as the covariance matrix of the changing level of occipital $\alpha$-activity. It should be mentioned that, to obtain this noise covariance matrix, additional EEG measurements must be performed to acquire the corresponding EEG signals and compute their covariance matrix. Since such measurements are not available for the EEG data sets analyzed here, iCSP will not be considered for evaluation in this paper. However, it still seems to be an efficient approach to make CSP robust against known noise sources.

\section{B. New RCSP algorithms}

In this section, we propose 4 new algorithms to regularize CSP: a CSP regularized with selected subjects, a Tikhonov Regularized CSP, a weighted Tikhonov Regularized CSP and a spatially Regularized CSP.

\section{1) Regularized CSP with Selected Subjects:}

This first new RCSP belongs to the same family as CCSP since it uses data from other subjects to shrink the covariance matrix towards a generic matrix $G_{c}$ (it uses $\beta \geq 0$ and $\alpha=$ $\gamma=0$ ). However, contrary to CCSP or GLRCSP, the proposed algorithm does not use the data from all available subjects but only from selected subjects. Indeed, even if data from many subjects is available, it may not be relevant to use all of them, due to potentially large inter-subject variabilities. Thus, we propose to build $G_{c}$ from the covariance matrices of a subset of selected subjects. We therefore denote this algorithm as RCSP with Selected Subjects or SSRCSP. With SSRCSP, the generic covariance matrix is defined as $G_{c}=\frac{1}{\left|S_{t}(\Omega)\right|} \sum_{i \in S_{t}(\Omega)} C_{c}^{i}$, where $|A|$ is the number of elements in set $A$ and $S_{t}(\Omega)$ is the subset of selected subjects from $\Omega$.

To select an appropriate subset of subjects $S_{t}(\Omega)$, we propose the subject selection algorithm described in Algorithm 1. In this algorithm, the function accuracy $=$ trainThenTest(trainingSet, testingSet) returns the accuracy obtained when training an SSRCSP with $\beta=1$ (i.e., using only data from other subjects) on the data set trainingSet and testing it on data set testingSet, with an LDA as classifier. The function $\left(\right.$ best $\left._{i}, \max _{f(i)}\right)=$ $\max _{i} f(i)$ returns best ${ }_{i}$, the value of $i$ for which $f(i)$ reaches its maximum $\max _{f(i)}$. In short, this algorithm sequentially selects the subject to add or to remove from the current subset of subjects, in order to maximize the accuracy obtained when training the $\mathrm{BCI}$ on the data from this subset of subjects and testing it on the training data of the target subject. This algorithm has the same structure as the Sequential Forward
Floating Search algorithm [18], used to select a relevant subset of features. This ensures the convergence of our algorithm as well as the selection of a good subset of additional subjects.

Input: $D_{t}$ : training EEG data from the target subject.

Input: $\Omega=\left\{D_{s}\right\}, s \in\left[0, N_{s}\right]$ : set of EEG data from the $N_{s}$ other subjects available $\left(D_{t} \ni \Omega\right)$.

Output: $S_{t}(\Omega)$ : a subset of relevant subjects whose data can be used to classify the data $D_{t}$ of the target subject.

selected $_{0}=\{\}$;

remaining $g_{0}=\Omega$

accuracy $_{0}=0 ; n=1$

while $n<N_{s}$ do

Step 1: (bestSubject, bestAccuracy) = max $_{s \in \text { remaining }_{n-1}}$ trainThenTest(selected ${ }_{n-1}+$ $\left.\left\{D_{s}\right\}, D_{t}\right)$;

selected $_{n}=$ selected $_{n-1}+\left\{D_{\text {bestSubject }}\right\}$ remaining $_{n}=$ remaining $_{n-1}-\left\{D_{\text {bestSubject }}\right\}$; accuracy $_{n}=$ bestAccuracy;

$n=n+1$

Step 2: if $n>2$ then

(bestSubject, bestAccuracy) $=$

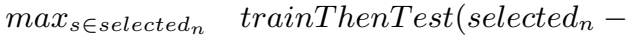
$\left.\left\{D_{s}\right\}, D_{t}\right)$

if bestAccuracy $>$ accuracy $_{n-1}$ then selected $_{n-1}=$ selected $_{n}-\left\{D_{\text {bestSubject }}\right\}$; remaining $_{n-1}=$ remaining $_{n}+\left\{D_{\text {bestSubject }}\right\}$; accuracy $_{n-1}=$ bestAccuracy; $n=n-1$ go to Step 2;

else go to Step 1; end end

end

$($ best $N$, selectedAcc $)=\max _{n \in\left[1, N_{s}\right] \quad \text { accuracy }_{n}}$; $S_{t}(\Omega)=$ selected $_{\text {best } N}$;

Algorithm 1: Subject selection algorithm for the SSRCSP (Regularized CSP with Selected Subjects) algorithm.

\section{2) CSP with Tikhonov Regularization:}

The next new algorithms we propose are based on the regularization of the CSP objective function using quadratic penalties (with $\alpha \geq 0, \gamma=\beta=0$ and $s_{c}=1$ ). The first one is a CSP with Tikhonov Regularization (TR) or TRCSP. Tikhonov Regularization is a classical form of regularization, initially introduced for regression problems [19], and which consists in penalizing solutions with large weights. The penalty term is then $P(w)=\|w\|^{2}=w^{T} w=w^{T} I w$. TRCSP is then simply obtained by using $K=I$ in the proposed framework (see Table I). Such regularization is expected to constrain the solution to filters with a small norm, hence mitigating the influence of artifacts and outliers.

3) CSP with Weighted Tikhonov Regularization:

With TRCSP, high weights are penalized equally for each channel. However, we know that some channels are more important than others to classify a given mental state. Thus, it may be interesting to have different penalties for different channels. If we believe that a channel is unlikely to have a large contribution in the spatial filters, then we should give it a relatively large penalty, in order to prevent CSP from assigning 
it a large contribution (which can happen due to artifacts for instance). On the other hand, if a channel is likely to be useful, we should not prevent CSP from giving it high weights, as this channel is likely to have a genuinely large contribution.

Formally, this leads to a penalty term of the form $P(w)=$ $w^{T} D_{w} w$, where $D_{w}$ is a diagonal matrix such that $D_{w}=$ $\operatorname{diag}\left(w_{G}\right)$ and $w_{G}(i)$ is the level of penalty assigned to channel $i$. Weighted Tikhonov Regularized CSP (WTRCSP) is then obtained by using $K=D_{w}$. These penalty levels $w_{G}(i)$ can be defined according to the literature, i.e., according to which brain regions (and thus channels) are expected to be useful. However, it may be difficult to select manually an appropriate penalty value for each channel. In this paper, we therefore use data from other subjects to obtain $w_{G}$ :

$$
w_{G}=\left(\frac{1}{2 \times N_{f} \times|\Omega|} \sum_{i \in \Omega} \sum_{f=1}^{2 \times N_{f}}\left|\frac{w_{f}^{i}}{\left\|w_{f}^{i}\right\|}\right|\right)^{-1}
$$

where $w_{f}^{i}$ is the $f^{t h}$ spatial filter obtained using CSP (among the eigenvectors corresponding to the $N_{f}$ largest and lowest eigenvalues of $M$, see Table I) for the $i^{t h}$ additional subject available. In other words, the penalty level of a channel is set to the inverse of the average absolute value of the normalized weight of this channel in the CSP filters obtained from other subjects (the less important the average channel weight, the higher the penalty). By doing so, we expect that the degree of usefulness of a given channel would be reflected by its weight in the filters obtained with CSP from other subjects.

\section{4) Spatially Regularized CSP:}

The last algorithm we propose is a Spatially Regularized CSP (SRCSP). The motivation behind this algorithm is that despite being used to learn spatial filters, CSP completely ignores the spatial location of EEG electrodes. SRCSP aims at making use of this spatial information. More particularly, we would like to obtain spatially smooth filters $w$, i.e, filters for which neighboring electrodes have relatively similar weights. Indeed, from a neurophysiological point of view, neighboring neurons tend to have similar functions, which supports the idea that neighboring electrodes should measure similar brain signals (if they are close enough to each other). To ensure spatial smoothness of the filters $w$, we use a Laplacian penalty term $P(w)$ as in [20], with the following regularization matrix $K$ :

$$
K=D_{G}-G \quad \text { with } \quad G(i, j)=\exp \left(-\frac{1}{2} \frac{\left\|v_{i}-v_{j}\right\|^{2}}{r^{2}}\right)
$$

where $v_{i}$ is the vector containing the $3 \mathrm{D}$ coordinates of the $i^{t h}$ electrode, and $D_{G}$ is a diagonal matrix such as $D_{G}(i, i)=$ $\sum_{j} G(i, j)$. Here, $r$ is a hyperparameter which defines how far two electrodes can be to be still considered as close to each other. As $w^{T}\left(D_{G}-G\right) w=\sum_{i, j} G(i, j)\left(w_{i}-w_{j}\right)^{2}$ (see, e.g., [21]), the penalty term $P(w)=w^{T} K w$ will be large for nonsmooth filters, i.e., for filters in which neighboring electrodes have very different weights.

\section{Hyperparameter selection}

All RCSP algorithms presented here (expect DLCSPauto) have one or more regularization parameters whose value must be defined by the user: $\alpha, \beta$ and $\gamma$ (see Table I). SRCSP has also its own specific hyperparameter: $r$ which defines the size of the neighborhood considered for smoothing. In [10] and [11], the selection of the regularization parameters for GLRCSP and CCSP was not addressed, and the authors presented the results for several values of the hyperparameters. In this paper, we used cross-validation (CV) to select these values. More precisely, we used as optimal hyperparameter values, those that maximized the 10 -fold cross validation accuracy on the training set by using LDA [2] as classifier. We selected values among the set $[0,0.1,0.2, \ldots, 0.9]$ for the parameters $\beta$ and $\gamma$, among the set $\left[10^{-10}, 10^{-9}, \ldots, 10^{-1}\right]$ for $\alpha$, and among $[0.01,0.05,0.1,0.5,0.8,1.0,1.2,1.5]$ for $r$.

\section{The best of two worlds?}

So far, all the algorithms presented use a single form of regularization: either they regularize the covariance matrices or the objective function, but not both. However, it is easy to imagine an algorithm combining these two approaches. We evaluated some such algorithms, e.g., a SRCSP with Diagonal Loading or a TRCSP with Generic Learning, among others. Unfortunately, none of them reached performances as high as that of the corresponding RCSP with a single form of regularization (results not reported here due to space limitations). Thus, it seems that RCSP with a single form of regularization are the simplest and most efficient algorithms.

\section{Evaluation}

\section{A. EEG data sets used for evaluation}

In order to assess and compare the RCSP algorithms presented here, we used EEG data from 17 subjects, from 3 publicly available data sets of BCI competitions. These three data sets contain Motor Imagery (MI) EEG signals, i.e., EEG signals recorded while subjects imagine limb movements (e.g., hand or foot movements) [1]. They are described below.

1) Data set IVa, BCI competition III: Data set IVa [22], from BCI competition III [6], contains EEG signals from 5 subjects, who performed right hand and foot MI. EEG were recorded using 118 electrodes. A training set and a testing set were available for each subject. Their size was different for each subject. More precisely, 280 trials were available for each subject, among which 168, 224, 84, 56 and 28 composed the training set for subject A1, A2, A3, A4 and A5 respectively, the remaining trials composing their test set.

2) Data set IIIa, BCI competition III: Data set IIIa [23], from BCI competition III [6], comprises EEG signals from 3 subjects who performed left hand, right hand, foot and tongue MI. EEG signals were recorded using 60 electrodes. For the purpose of this study, only EEG signals corresponding to left and right hand $\mathrm{MI}$ were used. A training and a testing set were available for each subject. Both sets contain 45 trials per class for subject B1, and 30 trials per class for subjects B2 and B3.

3) Data set IIa, BCI competition IV: Data set IIa [24], from BCI competition IV ${ }^{1}$ comprises EEG signals from 9 subjects who performed left hand, right hand, foot and tongue MI. EEG

\footnotetext{
${ }^{1}$ http://www.bbci.de/competition/iv/
} 
signals were recorded using 22 electrodes. Only EEG signals corresponding to left and right hand MI were used for the present study. A training and a testing set were available for each subject, both sets containing 72 trials for each class.

\section{B. Preprocessing}

In this work, we considered the discrete classification of the trials, i.e., we assigned a class to each trial. For each data set and trial, we extracted features from the time segment located from $0.5 \mathrm{~s}$ to $2.5 \mathrm{~s}$ after the cue instructing the subject to perform MI (as done by the winner of BCI competition IV, data set IIa). Each trial was band-pass filtered in $8-30 \mathrm{~Hz}$, as in [3], using a $5^{\text {th }}$ order Butterworth filter. With each (R)CSP algorithm, we used 3 pairs of filters for feature extraction $\left(N_{f}=3\right)$, as recommended in [4].

\section{Results and discussion}

For each subject, the (R)CSP filters were learnt on the training set available. The log-variances of the spatially filtered EEG signals were then used as input features to an LDA, one of the most efficient classifiers for BCI [2]. Table II reports on the classification accuracies obtained on the test sets.

Results show that, except DLCSPauto and CCSP2, all RCSP algorithms outperformed classical CSP, often substantially. The best RCSP algorithms outperformed CSP by about 3 to $4 \%$ in mean classification accuracy and by almost $10 \%$ in median classification accuracy. This confirms that when using CSP, regularization should be used in order to deal with its non-robust nature. The performance variance is also lower for RCSP algorithms. This, together with a closer look at the results, suggests that RCSP algorithms are more valuable for subjects with poor initial performances (e.g., A3, A5, B2, C5), than for already good subjects, whose performances are roughly unchanged. This makes sense as regularization aims at dealing with noisy or limited data, but not necessarily at improving performances for already good and clean data.

The best RCSP algorithm on these data is the WTRCSP that we proposed in this paper, as it reached both the highest median and mean accuracy. It is only slightly better than TRCSP, also proposed in this paper. These two algorithms have only a single hyperparameter to tune $(\alpha)$, which makes them more convenient to use and more computationally efficient than other good RCSP algorithms such as GLRCSP or SRCSP, which both have two hyperparameters.

In terms of statistical significance, a Friedman test [25][26] revealed that the RCSP algorithm used had a significant effect on the classification performance, at the $5 \%$ level $(p=0.03)$. It should be noted that we used the Friedman test because it is a non-parametric equivalent of the repeated measure ANOVA. Indeed, the fact that the mean and median accuracies are rather different in our data suggests that the accuracy may not be normally distributed. This makes the use of ANOVA inappropriate [25]. Post-hoc multiple comparisons revealed that TRCSP is significantly more efficient than CSP and DLCSPcvdiff. Actually, TRCSP appeared as the only algorithm which is significantly more efficient than CSP. However, other RCSP also seem more efficient than CSP but perhaps not significantly so due to the relatively modest sample size. Both TRCSP and WTRCSP appeared as significantly more efficient than GLRCSP, CCSP1, CCSP2 and DLCSPcv. Finally, SRCSP was significantly more efficient than CCSP1 and DLCSPcv.

In general, regularizing the CSP objective function seems more rewarding, in terms of performances, than regularizing the covariance matrices. A possible explanation might be found in Vapnik's statistical learning theory which advocates that "when solving a given problem, try to avoid solving a more generic problem as an intermediate step" [27]. Indeed, the objective of RCSP algorithms is to promote the learning of good spatial filters. However, when using covariance matrix regularization, we try to solve the more generic intermediate problem of obtaining a good covariance matrix estimate. We then hope this will lead to better spatial filters even though this was not directly addressed. On the other hand, when regularizing the objective function, we directly try to learn better spatial filters by enforcing some good prior structures for these filters. This might be an explanation as to why the latter approach is generally more efficient than the former.

Results obtained by CCSP, GLRCSP, SSRCSP and WTRCSP showed that subject-to-subject transfer in BCI is possible and valuable. Despite large inter-subject variabilities, knowing that using data from other subjects can improve performances may also benefit other EEG signal processing algorithms. Among RCSP methods using only data from other subjects to regularize the covariance matrices (i.e., CCSP1, CCSP2 and SSRCSP), SSRCSP reached the highest mean accuracy, which suggests that it is worth selecting subjects to build $G_{c}$. However, overall, WTRCSP appears as the most efficient algorithm that exploits subject-to-subject transfer. Nevertheless, since RCSP algorithms based on subject-tosubject transfer require EEG data from additional subjects, it may not always be possible to use them. Indeed, the performance improvement they offer may not justify the additional time required to collect data from new subjects. However, if data from other subjects is already available, which is likely to be the case if a given BCI system has been used for some times, then such RCSP algorithms are worth being used. Moreover, our study used a rather small number of additional subjects ( 2 to 8 depending on the data set). With a larger data base of additional subjects, performance improvements due to subject-to-subject transfer may be even larger (see, e.g., [28]).

Concerning the poor performances of DLCSPauto, it should be mentioned that we also observed poor performance when all training data were used in one of our previous studies [13]. However, when using this approach with a small training set, DLCSPauto proved to be significantly more efficient than CSP. This suggests DLCSPauto is most useful when very little training data is available. A comparison of DLCSPcv with DLCSPcvdiff showed that they obtained very similar performances. Actually, for 13 subjects out of 17, DLCSPcvdiff selected the same value for the regularization parameters of $C_{1}$ and $C_{2}$, i.e., it was equivalent to DLCSPcv. This suggests that it may not be necessary to use different regularization parameter values for each covariance matrix.

The fact that RCSP algorithms led to lower scores than CSP on a few subjects is also surprising. Indeed, if the best 
TABLE II

CLASSIFICATION ACCURACIES (MEAN, MEDIAN AND STANDARD DEVIATION (STD) IN \%) OBTAINED FOR EACH SUBJECT FOR THE STANDARD CSP AND THE REgULARIZED CSP ALGORITHMS PRESENTED IN THIS PAPER. FOR EACH SUBJECT, THE BEST RESULT IS DISPLAYED IN BOLD CHARACTERS.

\begin{tabular}{|c|c|c|c|c|c|c|c|c|c|c|c|c|c|c|c|c|c|c|c|c|}
\hline & \multicolumn{8}{|c|}{ BCI competition III } & \multicolumn{9}{|c|}{ BCI competition IV } & \multirow{2}{*}{\multicolumn{3}{|c|}{ Overall }} \\
\hline & \multicolumn{5}{|c|}{ data set IVa } & \multicolumn{3}{|c|}{ data set IIIa } & \multicolumn{9}{|c|}{ data set IIa } & & & \\
\hline & A1 & A2 & A3 & A4 & A5 & B1 & B2 & B3 & $\mathrm{C} 1$ & $\mathrm{C} 2$ & $\mathrm{C} 3$ & $\mathrm{C} 4$ & $\mathrm{C} 5$ & C6 & $\mathrm{C} 7$ & $\mathrm{C} 8$ & C9 & Mean & Median & $\mathrm{td}$ \\
\hline CSP & 66.07 & 96.43 & 47.45 & 71.88 & 49.6 & 95.56 & 61.67 & 93.33 & 88.89 & 51.39 & 96.53 & 70.14 & 54.86 & 71.53 & 81.25 & 93.75 & 3.75 & 75.5 & 71.9 & 18.2 \\
\hline$\overline{\mathrm{G}}$ & 2.32 & 3 & 66.84 & 67.86 & 89.29 & 5.56 & 61.67 & 90 & 5.11 & & 93.75 & 67.36 & .56 & 65.28 & 81.25 & 93.75 & & 3.2 & .3 & 4.3 \\
\hline & & & 7 & 71 & 8 & 39 & 4 & & & & & 94 & & & & & & 76.2 & .3 & 17.5 \\
\hline & & 5.43 & +1 & 71 & 49. & 56 & 61. & 93 & & & 2 & 70.14 & & 6 & 79.17 & 4 & & 75 & 1.9 & 18.3 \\
\hline & U & 5.43 & 94 & 71.43 & 50 & 4 & 6 & 9 & 9 & 9 & 53 & 70.14 & 4 & 1.53 & 1.94 & 75 & 93. & te & 5 & 18 \\
\hline & .29 & .43 & 52.04 & 71.88 & 82.54 & 5.56 & 78.33 & 93.3 & 39 & 0.69 & 6.53 & 70.14 & 56 & 62.5 & 1.25 & 93.75 & 86.81 & 77.7 & .3 & 16.1 \\
\hline $\mathrm{LC}$ & 69.64 & 98.21 & 55.1 & 71.88 & 82.54 & 95.56 & 66.67 & 93.3. & 3.89 & 50.69 & 6.53 & 70.14 & 55.56 & 62.5 & 81.25 & 93.75 & 5.81 & 77.6 & 1.3 & 16 \\
\hline SS & $\overline{0.54}$ & 96.43 & 53.57 & 71.88 & 75.39 & 95.56 & 61.67 & 96.67 & 8.89 & 53.47 & 97.22 & 70.14 & 56.25 & 68.75 & 79.17 & 97.22 & 90.28 & 77.8 & 5.4 & 16.2 \\
\hline & 43 & 43 & \begin{tabular}{|l|}
63.27 \\
\end{tabular} & 71.88 & 86.9 & 98.89 & 56.67 & 93.33 & 89 & 4.17 & 5.53 & 70.83 & 62.5 & 7.36 & 81.25 & 95.87 & 67 & 79.3 & 1.3 & 15.3 \\
\hline WTK & 69.64 & 98.21 & 54.59 & 71.88 & 85.32 & 98.89 & 71.67 & 93.33 & 88.89 & 54.86 & 96.53 & 70.14 & 65.97 & 61.81 & 81.25 & 95.83 & 90.97 & 79.4 & 81.3 & 15.3 \\
\hline SRCSP & 72.32 & 96.43 & 60.2 & 77.68 & 86.51 & 96.67 & 53.33 & 93.33 & $\mathbf{8 8 . 8 9}$ & 63.19 & 96.53 & 66.67 & 63.19 & 63.89 & 78.47 & 95.83 & 92.36 & $\overline{79.2}$ & 78.5 & 15.2 \\
\hline
\end{tabular}

results could be obtained without regularization, then we could expect that the hyperparameter selection procedure based on cross-validation would figure it out and set the regularization parameter to 0 . However, it sometimes seemed otherwise. This may suggest that, perhaps due to the non-stationarity of EEG, cross-validation is not a very good predictor of generalization performances for BCI. This has been also observed in one subject in [9]. We will investigate this issue in the future.

Figure 1 shows some examples of spatial filters obtained with different (R)CSP algorithms for different subjects. In general, these pictures show that CSP filters appear as messy, with large weights in several unexpected locations from a neurophysiological point of view. On the contrary, RCSP filters are generally smoother and physiologically more relevant, with strong weights over the motor cortex areas, as expected from the literature [1]. This suggests that another benefit of RCSP algorithms is to lead to filters that are neurophysiologically more plausible and as such more interpretable.

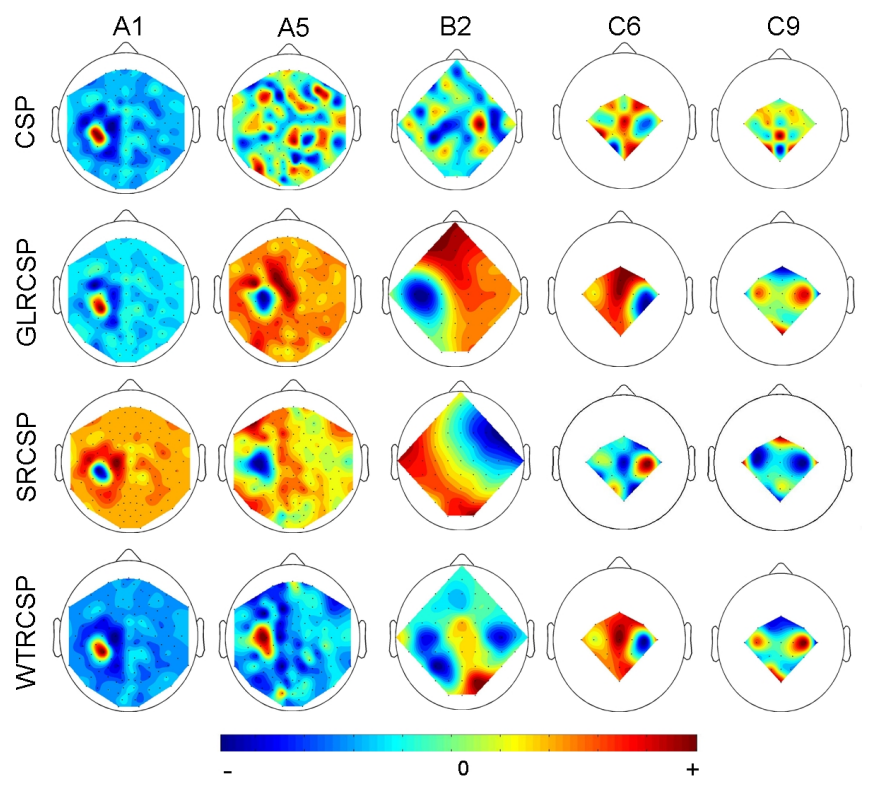

Fig. 1. Electrode weights for corresponding filters obtained with different CSP algorithms (CSP, GLRCSP, SRCSP and WTRCSP), for subjects A1, A5 (118 electrodes), B2 (60 electrodes) and C6, C9 (22 electrodes).

\section{CONCLUSION}

In this paper, we proposed a unified theoretical framework to design Regularized CSP. We proposed a review of existing RCSP algorithms, and presented how to cast them in this framework. We also proposed 4 new RCSP algorithms. We evaluated 11 different RCSP algorithms (including the 4 new ones and the original CSP), on EEG data from 17 subjects, from BCI competition data sets. Results showed that the best RCSP can outperform CSP by almost 10\% in median classification accuracy and lead to more neurophysiologically relevant spatial filters. They also showed that RCSP can perform efficient subject-to-subject transfer. Overall, the best RCSP on these data were WTRCSP and TRCSP, both newly proposed in this paper. Therefore, we would recommend BCI designers using CSP to adopt RCSP algorithms in order to obtain more robust systems. To encourage such an adoption and to ensure the present study replicability, a Matlab toolbox with all CSP algorithms evaluated in this paper is freely available upon request to the authors (e-mail: fabien.lotte@gmail.com).

Future work could deal with investigating performances of RCSP algorithms with very small training sets, so as to reduce BCI calibration time, in the line of our previous studies [13][29]. It could also be interesting to adapt the presented regularization framework to multiclass CSP approaches based on approximate joint diagonalization such as [30]. We could also cast the problem of subject-to-subject transfer for RCSP as a multitask problem. In this case, the mean and/or variance of spatial filters learnt across multiple subjects would be used as prior information, in a similar flavor as what has been done in [31] for learning linear classifiers. Finally, we could explore the integration of the regularization terms proposed here into one-step procedures, which learn the spatial filter and the classifier simultaneously, as in [32].

Acknowledgments: The authors would like to thank Dr. Schlögl and Dr. Blankertz for providing the electrode coordinates of BCI competition data, and Dr. Hamadicharef, Ms. Rosendale and anonymous reviewers for their constructive comments. 


\section{REFERENCES}

[1] G. Pfurtscheller and C. Neuper, "Motor imagery and direct braincomputer communication," proc IEEE, vol. 89, no. 7, pp. 1123-1134, 2001.

[2] F. Lotte, M. Congedo, A. Lécuyer, F. Lamarche, and B. Arnaldi, "A review of classification algorithms for EEG-based brain-computer interfaces," J Neural Eng, vol. 4, pp. R1-R13, 2007.

[3] H. Ramoser, J. Muller-Gerking, and G. Pfurtscheller, "Optimal spatial filtering of single trial EEG during imagined hand movement," IEEE Trans Rehab Eng, vol. 8, no. 4, pp. 441-446, 2000.

[4] B. Blankertz, R. Tomioka, S. Lemm, M. Kawanabe, and K.-R. Müller, "Optimizing spatial filters for robust EEG single-trial analysis," IEEE Signal Proc Magazine, vol. 25, no. 1, pp. 41-56, 2008.

[5] B. Blankertz, K. R. Müller, G. Curio, T. M. Vaughan, G. Schalk, J. R. Wolpaw, A. Schlögl, C. Neuper, G. Pfurtscheller, T. Hinterberger, M. Schröder, and N. Birbaumer, "The BCI competition 2003: Progress and perspectives in detection and discrimination of EEG single trials," IEEE Trans Biomed Eng, vol. 51, no. 6, pp. 1044-1051, 2004.

[6] B. Blankertz, K. R. Müller, D. J. Krusienski, G. Schalk, J. R. Wolpaw, A. Schlögl, G. Pfurtscheller, J. D. R. Míllan, M. Schroder, and N. Birbaumer, "The BCI competition III: Validating alternative approaches to actual BCI problems," IEEE Trans Neural Syst Rehab, vol. 14, no. 2, pp. 153-159, 2006.

[7] B. Reuderink and M. Poel, "Robustness of the common spatial patterns algorithm in the BCI-pipeline," University of Twente, Tech. Rep., 2008.

[8] M. Grosse-Wentrup, C. Liefhold, K. Gramann, and M. Buss, "Beamforming in non invasive brain computer interfaces," IEEE Trans Biomed Eng, vol. 56, no. 4, pp. 1209 - 1219, 2009.

[9] B. Blankertz, M. Kawanabe, R. Tomioka, F. Hohlefeld, V. Nikulin, and K.-R. Müller, "Invariant common spatial patterns: Alleviating nonstationarities in brain-computer interfacing," in NIPS 20, 2008.

[10] H. Lu, K. Plataniotis, and A. Venetsanopoulos, "Regularized common spatial patterns with generic learning for EEG signal classification," in $E M B C, 2009$, pp. $6599-6602$.

[11] H. Kang, Y. Nam, and S. Choi, "Composite common spatial pattern for subject-to-subject transfer," IEEE Sig Proc Let, vol. 16, no. 8, pp. 683 $-686,2009$.

[12] F. Lotte and C. Guan, "Spatially regularized common spatial patterns for EEG classification," in ICPR, 2010, pp. 3712-3715.

[13] — , "Learning from other subjects helps reducing brain-computer interface calibration time," in ICASSP, 2010, pp. 614-617.

[14] J. Farquhar, N. Hill, T. Lal, and B. Schölkopf, "Regularised CSP for sensor selection in BCI," in 3rd international BCI workshop, 2006.

[15] X. Yong, R. Ward, and G. Birch, "Sparse spatial filter optimization for EEG channel reduction in brain-computer interface," in ICASSP, 2008, pp. 417-420.

[16] O. Ledoit and M. Wolf, "A well-conditioned estimator for largedimensional covariance matrices," J Multivariate Analysis, vol. 88, no. 2, pp. 365-411, 2004.

[17] C. Vidaurre, N. Krämer, B. Blankertz, and A. Schlögl, "Time domain parameters as a feature for EEG-based brain computer interfaces," Neural Networks, vol. 22, pp. 1313-1319, 2009.

[18] P. Pudil, F. J. Ferri, and J. Kittler, "Floating search methods for feature selection with non monotonic criterion functions," Patt Recog, vol. 2 pp. 279-283, 1994.

[19] A. Tikhonov, "Regularization of incorrectly posed problems," Soviet Math., vol. 4, pp. 1624-1627, 1963.

[20] Z. Xiang, Y. Xi, U. Hasson, and P. Ramadge, "Boosting with spatial regularization," in NIPS, 2009.

[21] D. Cai, X. He, Y. Hu, J. Han, and T. Huang, "Learning a spatially smooth subspace for face recognition," in CVPR, 2007.

[22] G. Dornhege, B. Blankertz, G. Curio, and K. Müller, "Boosting bit rates in non-invasive EEG single-trial classifications by feature combination and multi-class paradigms," IEEE Trans Biomed Eng, vol. 51, no. 6, pp. 993-1002, 2004.

[23] A. Schlögl, F. Lee, H. Bischof, and G. Pfurtscheller, "Characterization of four-class motor imagery EEG data for the BCI-competition 2005," J Neural Eng, pp. L14-L22, 2005.

[24] M. Naeem, C. Brunner, R. Leeb, B. Graimann, and G. Pfurtscheller, "Seperability of four-class motor imagery data using independent components analysis," J Neural Eng, vol. 3, pp. 208-216, 2006.

[25] P. Sprent and N. Smeeton, Applied nonparametric statistical methods. Chapman \& Hall, 2001.

[26] G. Cardillo. (2009) Myfriedman: Friedman test for non parametric repeated measure analysis of variance. [Online]. Available: http: //www.mathworks.com/matlabcentral/fileexchange/25882
[27] V. Vapnik, The Nature of Statistical Learning Theory, Second edition. Springer-Verlag, New-York, 2000.

[28] S. Fazli, F. Popescu, M. Danóczy, B. Blankertz, K.-R. Müller, and C. Grozea, "Subject-independent mental state classification in single trials," Neural Networks, vol. 22, no. 9, pp. 1305-1312, 2009.

[29] F. Lotte and C. Guan, "An efficient P300-based brain-computer interface with minimal calibration time," in AMD-NIPS, 2009.

[30] M. Grosse-Wentrup and M. Buss, "Multi-class common spatial pattern and information theoretic feature extraction," IEEE Trans on Biomed Eng, vol. 55, no. 8, pp. 1991-2000, 2008.

[31] M. Alamgir, M. Grosse-Wentrup, and Y. Altun, "Multitask learning for brain-computer interfaces," in AISTATS, 2010, pp. 17-24.

[32] R. Tomioka and K.-R. Müller, "A regularized discriminative framework for EEG analysis with application to brain-computer interface," $\mathrm{Neu}$ roimage, vol. 49, no. 1, pp. 415-432, 2010.

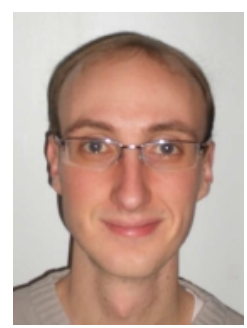

Fabien LOTTE obtained a M.Sc., a M.Eng. and a $\mathrm{PhD}$ degree in computer sciences, all from the $\mathrm{Na}$ tional Institute of Applied Sciences (INSA) Rennes, France, in 2005 and 2008 respectively. As a PhD candidate he was part of the French National Research Institute for Computer Science and Control (INRIA) and was supervised by Dr. Anatole LECUYER and Pr. Bruno ARNALDI. His PhD Thesis received both the $\mathrm{PhD}$ Thesis award 2009 from AFRIF (French Association for Pattern Recognition) and the $\mathrm{PhD}$ Thesis award 2009 accessit (2nd prize) from ASTI (French Association for Information Sciences and Technologies). In October and November 2008, he was a visiting PhD student at the HIROSETANIKAWA laboratory, the University of Tokyo, Japan. Since January 2009, he works as a research fellow in Singapore, at the Institute for Infocomm Research (I2R), Signal processing department, in the Brain-Computer Interface laboratory lead by Dr. Cuntai GUAN. His research interests include braincomputer interfaces, pattern recognition, virtual reality and signal processing.

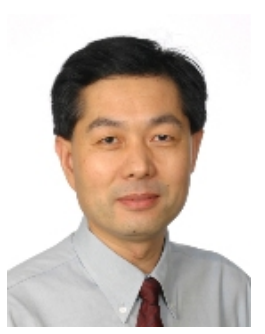

Cuntai GUAN is a Principal Scientist \& Program Manager at Institute for Infocomm Research, Agency for Science, Technology and Research, Singapore. He received the Ph.D. degree in electrical and electronic engineering from Southeast University, China, in 1993. From 1993 to 1994, he was at the Southeast University, where he worked on speech vocoder, speech recognition, and text-to-speech. In 1995, he was a Visiting Scientist at the Centre de Recherche en Informatique de Nancy, France, where he was working on key word spotting. From 1996 to 1997 , he was with the City University of Hong Kong, where he was developing robust speech recognition under noisy environment. From 1997 to 1999, he was with the Kent Ridge Digital Laboratories, Singapore, where he was working on multilingual, large vocabulary, continuous speech recognition. He was then a Research Manager and the R\&D Director for five years in industries, focusing on the research and development of spoken dialogue technologies. Since 2003, he established the Brain-computer Interface Laboratory at Institute for Infocomm Research. He has published over 90 refereed journal and conference papers, and holds 8 granted patents and applications. $\mathrm{He}$ is Associate Editor of Frontiers in Neuroprosthetics. He is a Senior Member of the IEEE, and President of Pattern Recognition and Machine Intelligence Association (PREMIA), Singapore. His current research interests include brain-computer interface, neural signal processing, machine learning, pattern classification, and statistical signal processing, with applications to neuro-rehabilitation, health monitoring, and cognitive training. 